\title{
Event-related potentials in clinical research: guidelines for eliciting, recording, and quantifying Mismatch Negativity, P300, and N400
}

\author{
Potenciais relacionados a eventos em pesquisa clínica: \\ diretrizes para eliciar, gravar, e quantificar o MMN, P300 \\ e N400
}

Event-Related Potentials (ERP) refer to electrical responses generated by the thalamus, auditory cortex and cortical association areas and involve discrimination, integration and attention tasks. Furthermore, they consist of a series of positive and negative peaks which take place from $50 \mathrm{~ms}$ after the stimulus onset and can be useful in investigating neural mechanisms related to auditory perception ${ }^{(1,2)}$.

The objective was to discuss the use of Event-Related Potentials (ERP) in clinical research for psychiatric and neurological disorders.

Techniques for obtaining, recording and analyzing P300, MMN and N-400 responses are presented in the article, especially the application of each of these potentials in the central nervous system pathology research.

The authors concentrate efforts on providing subsidies to the recommendation, clear and secure standardization of used methodologies, in order to facilitate comparison between laboratories, and strengthen research in the area. Therefore, it is relevant material for Brazilian researchers interested in using these methods.

ERP reflect the reception and processing of sensory information related to selective attention, memory upgrade, semantic understanding and other cognitive activities ${ }^{(3)}$. The latency informs course time of processing activity in milliseconds and the amplitude indicates the extent of neural allocation of resources involved in cognitive processes.

Mismatch negativity (MMN), one of its components, is elicited by any discriminated change in auditory stimulation and can be obtained in the absence of the participant's attention, which makes it useful in evaluating children or adults with cognitive and linguistic changes, such as schizophrenia, Alzheimer's, cerebrovascular accident, dyslexia and monitoring of coma.

This potential is generated from bilateral auditory cortex, with contributions of the right frontal cortex, and extracted from the discrepancy of acoustic characteristics of two sounds. It is recorded from the waveform difference, obtained by subtracting the standard stimulus mean from the deviant stimulus mean. For the record, in general, we use 5-10 active electrodes positioned at Fz, Cz, C3, C4, and mastoid, with the reference electrode placed on the nose.

Another potential, $\mathrm{P} 300$, is used to investigate conditions in which cognition is impaired and it is mostly used in clinical research since it informs on discrimination of stimuli when a process is active and operating. It has proven useful in the assessment of dementia, schizophrenia, psychiatric disorders, attention deficit disorder and hyperactivity and dyslexia.

P300 is a high and broad positive component with peaks of $300 \mathrm{~ms}$ or over, after the onset of a deviant stimulus amid a series of frequent stimuli. It has parieto-central distribution on the scalp and electrodes at Fz, $\mathrm{Cz}$ and $\mathrm{Pz}$, with reference to the earlobe or mastoid process. 
$\mathrm{N} 400$, the last component described in this paper is a negative component, which reaches its peak of amplitude approximately $400 \mathrm{~ms}$ after onset of stimulation. It is widely distributed on the scalp, with maximum amplitudes in the midline and parietal and lower amplitudes in frontal and lateral sites. This potential is generated in the left temporal lobe, with small contribution of the right temporal lobe, and its distribution seems to be more frontal in children than in adults.

The set of recording comprises $\mathrm{Fpz}, \mathrm{Fz}, \mathrm{Cz}, \mathrm{Pz}, \mathrm{Oz}$ and two lateral pairs on the anterior and posterior temporal lobe. Furthermore, horizontal and vertical visual recordings must be made.

N400 has been used to examine sensitivity to lexical and contextual characteristics and the neural substrates for the language. Moreover, it is promising to get answers about the specific nature of cognitive disorders, since it is a noninvasive tool and therefore used in patients with learning disorders and dyslexia.

According to the authors, clinical participants should be selected according to clear and well established diagnostic criteria, and samples should be as homogeneous as possible ${ }^{(1)}$. The literature suggests that the controls selected in the studies should differ from the clinical group only regarding the variable of interest and the drugs used by the subjects should be documented. It is also emphasized that there are highly significant, robust and replicable differences between clinical group and control group subjects. However, it is noteworthy that the variability involved in the measure still does not allow a precise and generalized classification, and therefore the usefulness of diagnosis of clinical disorders employing ERP is still limited.

On the other hand, studies with ERP need to be discussed and used in clinical practice in Brazil as the literature has shown the usefulness of this test for the investigation of several clinical disorders.

Ana Carla Leite Romero

Simone Fiuza Regacone

Daiane Damaris Baptista de Lima

Pedro de Lemos Menezes

Ana Cláudia Figueiredo Frizzo

Graduate Program in Audiology/Speech Patology, School of Philosophy and Sciences, Universidade Estadual Paulista Paulista "Júlio de Mesquita Filho" - UNESP - Marília (SP), Brazil and Universidade Estadual de Ciências da Saúde de Alagoas - UNCISAL - Maceió (AL), Brasil.

\section{REFERÊNCIAS}

1. Ventura LMP, Alvarenga KF, Costa Filho OA. Protocol to collect late latency auditory evoked potentials. Braz J Otorhinolaryngol. 2009;75(6):879-83. http://dx.doi.org/10.1590/S1808-86942009000600018

2. Zhang F, Anderson J, Samy R, Houston L. The adaptive pattern of the late auditory evoked potential elicited by repeated stimuli in cochlear implant users. Int J Audiol. 2010;49(4):277-85. http://dx.doi.org/10.3109/14992020903321759

3. Picton TW, Bentin S, Berg P, Donchin E, Hillyard SA, Johnson R Jr et al. Guidelines for using human event-related potentials to study cognition: recording standards and publication criteria. Psychophysiology. 2000;37(2):127-52. 